\title{
How to Make a Digital Currency on a Blockchain Stable
}

\author{
Kenji Saito ${ }^{\mathrm{a}, *}$, Mitsuru Iwamura ${ }^{\mathrm{b}}$ \\ ${ }^{a}$ Keio Research Institute at SFC, Keio University, (Murai Lab.) 5322 Endo, Fujisawa, \\ Kanagawa 252-0882 Japan \\ ${ }^{b}$ Graduate School of Business and Finance, Waseda University, 1-6-1 Nishi-Waseda, \\ Shinjuku-ku, Tokyo 169-8050 Japan
}

\begin{abstract}
Bitcoin and other similar digital currencies on blockchains are not ideal means for payment, because their prices tend to go up in the long term (thus people are incentivized to hoard those currencies), and to fluctuate widely in the short term (thus people would want to avoid risks of losing values).

The reason why those blockchain currencies based on proof of work are unstable may be found in their designs that the supplies of currencies do not respond to their positive and negative demand shocks, as the authors have formulated in our past work.

Continuing from our past work, this paper proposes minimal changes to the design of blockchain currencies so that their market prices are automatically stabilized, absorbing both positive and negative demand shocks of the currencies by autonomously controlling their supplies. Those changes are: 1) limiting re-adjustment of proof-of-work targets, 2) making mining rewards variable according to the observed over-threshold changes of block intervals, and 3) enforcing negative interests to remove old coins in circulation. We have made basic design checks and evaluations of these measures through simple simulations.

In addition to stabilization of prices, the proposed measures may have effects of making those currencies preferred means for payment by disincentivizing hoarding, and improving sustainability of the currency systems by making rewards to miners perpetual.
\end{abstract}

Keywords: digital currency, cryptocurrency, Bitcoin, blockchain, proof of work

\section{Introduction}

Digital currencies such as BTC on Bitcoin [1] blockchain and Ether on Ethereum[2] blockchain are now well-known, and are accepted means for payment in some

\footnotetext{
${ }^{*}$ Corresponding author.

Email addresses: ks91@sfc.wide.ad.jp (Kenji Saito), iwamuram@waseda.jp (Mitsuru Iwamura)
} 
countries. However, these currencies are not ideal as methods of payment because of instability of their market prices. Their prices tend to go up in the long term, and to fluctuate widely in the short term. Therefore, people are incentivized to hoard those currencies, and would want to avoid risks of losing values by using them for payment. Stability of their market prices needs to be achieved if these currencies are intended to be used as monetary media instead of as investment products.

In the authors' past work [3] (an improved version of the discussion paper with the same title), we have formulated the mechanism of instability by analyzing the economics of those blockchain currencies based on proof of work. We have also proposed measures to stabilize the market prices of such currencies.

The contributions of this paper are refinements of the work as follows:

1. We have refined the formulation of the economic model of blockchain currencies based on proof of work, using abstractions closer to actual currency system designs, and

2. We have refined our proposed measures for stabilization of market prices of the currencies that would absorb both positive and negative demand shocks.

The proposed measures went through basic design checks and evaluations by simple simulations.

Our proposals are primarily targeted for Bitcoin and its descendants. For information of the design of Bitcoin, in addition to the original design paper [1], we have referred to more up-to-date resources from the project web page 4 and the code1 itself.

\section{Blockchain Currency based on Proof of Work}

First, we clarify the design of blockchain currencies based on proof of work.

Any participant in the blockchain network may construct and broadcast (by flooding) a transaction of coins of which they have control. Figure 1 shows an example of transaction data structure found in Bitcoin. A transaction may have a list of inputs each referring to an existing coin as a container of value (represented as an output of a past transaction), and a non-empty list of outputs each representing a new coin or null coin (an output used for applications). A transaction is digitally signed in each input, which is verifiable with the public key also included in the input. The digest of the public key needs to match the digest to which the referred output is addressed. This structure is self-contained, and its validity can be verified by anyone.

The objective of the blockchain's consensus algorithm (Nakamoto consensus hereafter) is to maintain a single history of blocks such that it does not involve any invalid or contradicting transactions. An example of the latter is double spending of one digital coin, which cannot be prevented by the transaction data

\footnotetext{
${ }^{1}$ https://github.com/bitcoin/bitcoin/
} 


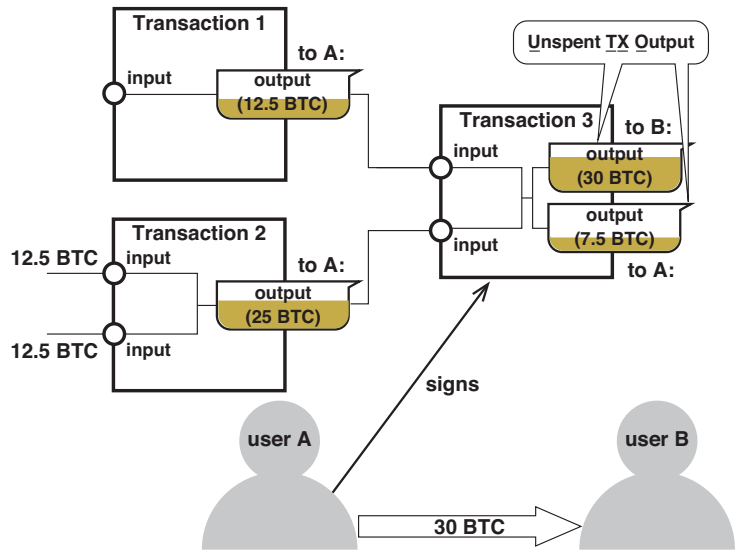

Figure 1: Transaction Structure of Bitcoin - So-called UTXO Model

structure alone. Presumably, the designer (or designers) of this technology first called it distributed timestamp server because it is intended to keep track of relative timing of events. As Figure 2 shows, each block contains the cryptographic digest of the previous block, except the very first block sometimes called the genesis block. Such a digest must meet a certain criterion; it needs to be less than or equal to the pre-adjusted and agreed target stored in or calculated from the block (we call this structure hash chain with proof of work hereafter). Since the digest is calculated by a one-way function whose outputs are evenly distributed, no one can intentionally configure a block to satisfy the criterion. Instead, they need to partake repetitive trials to change the values of some nonce in the block they are creating until they get a right digest. Therefore, creation of a block is a probabilistic process.

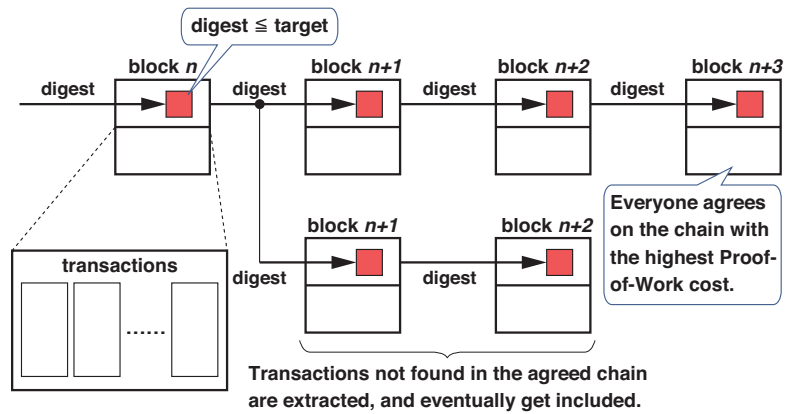

Figure 2: Overview of Bitcoin Blockchain - Hash Chain with Proof of Work

The necessity of repetitive trials functions as a proof-of-work mechanism intended to be a protection against falsification. A transaction itself cannot 
be falsified unless digital signatures are compromised. But it is conceivable to remove some transactions from a past block or to add fabricated transactions that did not really exist. If one tries so, the digest of the block is changed and is typically greater than the target. Then they would have to retry the proof of work for the block. This changes the digest stored in the next block, which in turn means that the digest of the next block is also changed and is typically greater than the target, and so on. In short, ones with a malicious intention would have to redo the proof of work from where they want to change, and outdo the ongoing process of adding blocks eventually to make the change valid, which has generally been considered highly difficult.

Such proof of work can also limit the number of proposed blocks at one time. But there still is a possibility of multiple participants each proposing a new block at roughly the same time, which may be accepted by different sets of participants. Then the hash chain may have multiple ends that are extended independently from one another, resulting in a fork of the blockchain with multiple (and possibly, contradicting) histories of blocks. If this happens, roughly speaking, the longest branch is considered to be correct. More precisely, to avoid the case of branches with artificially raised targets, the branch that is the most difficult to produce is chosen by all participants. This reflects the total cost cast in the creation of the hash chain branch. Because of proof of work, any chain branch requires the same cost paid for its creation when it is tried to be falsified. In short, Nakamoto consensus tries to enforce that the most difficult chain branch to falsify is chosen as the single correct history 2 .

\section{Market Price Mechanism of a Blockchain Currency based on Proof of Work}

\subsection{Supply and Demand}

The price of a blockchain currency and proof of work are closely related, because proof of work is closely related to supply of the coins, and the price in general is a reflection of supply and demand.

The authors have discussed this in detail in our past work [3] , and reasoned that the cause of instability of BTC price is that supply of coins does not respond to demand shocks.

Figure 3 shows the supply and demand curves of Bitcoin.

Usually for any goods (including money), demand curve slopes downwards from left to right (negatively sloped) while supply curve slopes positively. The price is found at the intersection of both curves. If the demand curve shifts left (demand drops) or right (demand rises), the supply curve would naturally respond (also shifts left to decreases supply or right to increase) to ease the shock of demand changes and subsequent price changes.

\footnotetext{
${ }^{2}$ For imperfection of the design of Nakamoto consensus, readers are referred to a past work [5] by the first author of this paper.
} 


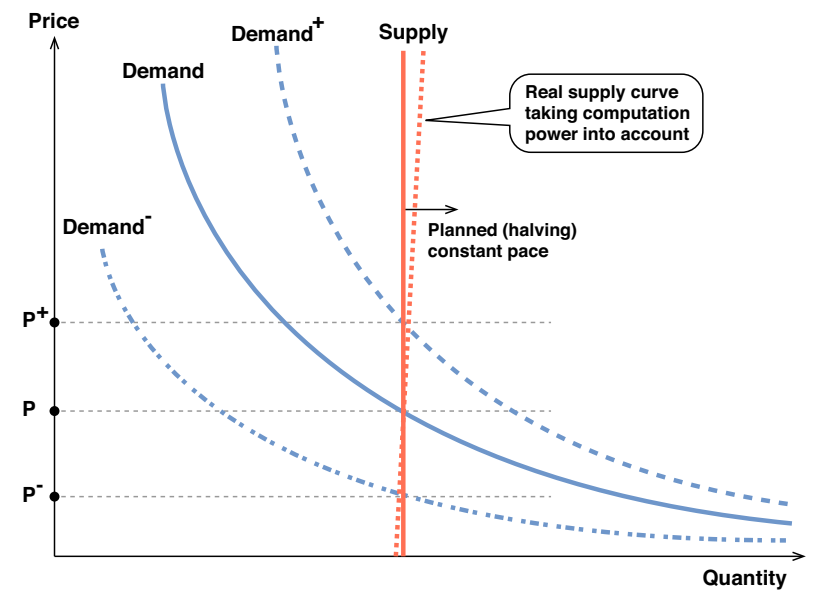

Figure 3: Supply and Demand of Bitcoin with a Vertical Supply Curve

On the other hand, BTC coins are only newly supplied as the reward in every new block. There just is a rule that the reward is halved every 210,000 blocks. Therefore the supply curve is vertical (more precisely, the supply curve is slightly positively sloped, because when the demand rises and the price goes up, there will be slightly more supply before the proof-of-work target is adjusted. When the demand drops and the price goes down, there will be the opposite effect). Shifting the demand curve directly affects the price $P$ in the figure.

\subsection{Consequences of Proof of Work}

Here, we present a revised simpler version of the formulation in our past work [3] to describe the economics of proof of work.

We start by the number of participants. Suppose $M$ miners participate. For simplicity, computation power of all miners are considered equal. Thus, $M$ is rather the total number of mining units with the same hash rate (how many digests can be calculated in an interval), and the number can increase or decrease to represent entry and exit of miners. Further, we assume that there is no monopoly in the mining industry, and miners' production function does not have externality or knowledge-intensiveness.

Usually, in the digital world, a production function is considered virtually free from the law of diminishing returns because the marginal cost of production is negligible. However, in the case of mining of a blockchain currency, because the proof-of-work target is adjusted to maintain the same production rate of the currency, adding more production units would at some point yield lower incremental per-unit returns, showing similar characteristics to productions in the physical world. For this reason and with above assumptions, we believe that the classical competition model (with entry and exit) can be applied to our arguments, as described below. 
Let $\lambda$ be the average number of occurrences of an event that a miner with one mining unit finds a nonce satisfying the required criterion in an interval. We ignore the cost of communication, so that discoveries of a right nonce are independent events that can globally occur $M \lambda$ times within the interval.

We consider the average interval between successes $\theta$ :

$$
\theta=\frac{1}{M \lambda}
$$

We assume a cryptographic hash function $H$ with range $R$. For example, in case of SHA-256, $R=2^{256}$. We define the proof-of-work criterion as follows: the cryptographic digest of a block by $H$ must be less than or equal to target $G$. We define target-reachability $g$ as below.

$$
g=\frac{G}{R}
$$

Conceptually, difficulty of proof of work is inverse of the target-reachability $g^{-1}$.

Suppose that one mining unit tries $h$ times in an interval, so that $h$ represents the average hash rate of a mining unit. Therefore,

$$
\lambda=h g
$$

and

$$
\theta=\frac{1}{M h g}
$$

Let $V$ be the reward of a successful proof of work. Then the reward for the winners of the mining competition is fixed as about $Z$ altogether per interval.

$$
Z=\frac{V}{\theta}=V M h g
$$

The expected benefit $B_{m}$ per mining unit per interval is therefore,

$$
B_{m}=\frac{Z}{M}=V h g
$$

If the market value of bitcoin is given as $P$, the market value of the expected benefit is $P B_{m}$.

Now, we consider the cost $C_{m}$ for operating one mining unit.

If $C_{m}<P B_{m}$, more mining units will participate ( $M$ is increased). This in turn will result in shorter $\theta$, and eventually $g$ will be adjusted with smaller $G$ to maintain the reference $\theta$ (10 minutes in the case of Bitcoin). This will result in $B_{m}$ getting smaller.

Conversely if $C_{m}>P B_{m}$, less mining units will participate ( $M$ is decreased). Short-term actualization of this would be that miners turn off their mining units. Long-term actualization would be that miners use their mining units to mine other compatible and profitable coins, or sell their units to others who mine 
such coins, or just dispose the units, thereby (partially) leaving from the mining business of the particular currency. Then $\theta$ is prolonged, and eventually $g$ will be adjusted with larger $G$, resulting in $B_{m}$ getting larger.

In the long run, the following equilibrium is reached.

$$
C_{m}=P B_{m}
$$

In other form,

$$
C_{m}=P V h g
$$

Some readers may wonder if such an equilibrium is still reached if the price $P$ goes down to zero. If $P$ stays at a near-zero level for a long time, the equation requires that the cost $C_{m}$ for operating one mining unit is very small. Please note that we can set $h$ (and subsequently $M$ ) at an arbitrary level, so that we can consider very small $h$ (like once in every 10 minutes) and very large $M$ to start with, where $C_{m}$ is then very small. In the case where $P$ is very low, $g$ approaches 1 as $M$ gets smaller and smaller, and in the end, the equilibrium would be reached. In reality, if $h$ is that infrequent, a miner can use some idle time of a computer used for other purposes, so that $C_{m}$ can actually be negligibly small ( $P$ being near zero is most likely caused by downturn of demands, so that by the time the near-zero-price situation arises, occurrences of transactions would become very sparse. Therefore the cost of verifying transactions can also be ignored). If $P$ is maintained to be zero for a long time, however, then the equation cannot hold, as $C_{m}$ is non-zero albeit it can be negligibly small. But if that really happens, then there is no economic reason for mining, so that no mining unit would want to participate, and eventually the blockchain would stop, in which case there is no point of discussing the equilibrium anyway.

In the case of Bitcoin, the right side of the equation is contributed as follows:

$P$ : Goes up and down mainly by speculation.

$V$ : Starts at 50BTC, and halved at every 210,000 blocks (12.5BTC as of year 2018).

$h$ : Increases by technological advance.

$g$ : Target $G$ is adjusted every 2,016 blocks to maintain the same reference value of $\theta$ (10 minutes).

The left side of the equation $C_{m}$, the cost for operating one mining unit, can be roughly modeled as $h$ multiplied by the unit cost of electricity $C_{m}^{e}$, so that $C_{m}=h C_{m}^{e}$. From the previous equation, we get $h C_{m}^{e}=P V h g$. By dividing both sides with $h$, we get $C_{m}^{e}=P V g$. This suggests that expected gain, $P V g$, will in long term balance with the unit cost of electricity $C_{m}^{e}$. In a long run, $C_{m}^{e}$ decreases while $h$ increases, both by technological advances, which are possibly motivated by rise of $P V g$. But their changes are gradual. 
In short term, there is no doubt that $P$ is the driving force of disequilibrium and subsequent changes in the number of mining units $M$ and then targetreachability $g$, but $P$ may also be affected by $g$ through people's expectations, which is affected by $M$. If $g$ gets halved by $M$ becoming doubled, there is a room for $P$ to get doubled. When $V$ gets halved at every 210,000 blocks, if $g$ is unmoved, then $P$ would need to be doubled to reach the equilibrium (and miners have reasons to want that to happen). If it does not happen, miners would need to leave, so that $M$ gets halved, and in turn, $g$ becomes doubled.

Equation (11) gives the long-term target for equilibrium, which we believe would explain the overall movements of price of bitcoins. In short term, it seems that the price goes up and down by people's expectations or operational intentions of the price to go up or down. This has been made possible because supply does not respond against demand shocks to begin with - it is easy to control the price by demands. We expect that observed wide fluctuations of price in short term would be improved if a modification is made in the design of the currency system such that the supply responds against demand shocks.

\section{Measures against Positive Demand Shock}

\subsection{Limited Target Re-adjustments}

To mitigate the effects of positive demand shock, in our past work [3] , we have suggested no adjustment to target $G$ unless $\theta$ is very short.

The reason is that we would like to increase the supply of coins by increasing $Z$ when the demand increases. To do so, we can only increase $V$ or shorten $\theta$. What miners can do autonomously through their own decisions is to increase $M$ (by making more investments), and thereby shorten $\theta$.

But how short is very short, where adjustment of $G$ is eventually needed? To prevent the blockchain from accidental forks as described in [6], $\theta$ should have the minimum limit. Let it be $\theta_{\text {min }}$. $G$ should be adjusted well before $\theta$ reaches $\theta_{\min }$. So we will adjust $G$ when a certain threshold $\theta^{\prime}$ is reached where $\theta^{\prime}>\theta_{\min }$.

\subsection{Target Re-adjustments for Positive Shock}

We propose that $\theta^{\prime}$ shall be the half of the reference $\theta$ (10 minutes in the case of Bitcoin), where $G$ must get halved. At the same time, we double $V$ to maintain the same pace of the supply of coins.

To make this happen, the system needs to refer to real time, which had been considered difficult for blockchains where timestamps in blocks can be untrue. But now, we have the concept of MTP (Median Time Past; the median time of the 11 blocks in the past) as a standard measure for the purpose. We check the average interval between blocks using MTP, every after 100 blocks, for example.

In the case of Bitcoin, because the reference $\theta$ is 10 minutes, 100 blocks takes about 17 hours. When $\theta$ is halved, it can be detected within a half day or so. 


\section{Measures against Negative Demand Shock}

\subsection{Two-fold Propositions}

We propose the measures to mitigate the effects of negative demand shock in two fold:

1. No adjustment to target $G$ unless the maximal tolerable average interval $\theta_{\max }$ is reached.

2. Continuous removal of coins in circulation so that less total supply of coins can be achieved, not only new supply.

For the former, we propose the following adjustment rule for $G$ : when $20 \%$ of $M$ is observed to have left ( $\theta$ is observed to be $\frac{5}{4}$ times longer than the reference value), make $G \frac{5}{4}$ times larger. At the same time, make $V 80 \%$ of the reward at the time to maintain the same pace of supply of coins. This adjustment will likely to occur within a couple of days after a negative demand shock. We think that this asymmetry in the rules against positive and negative demand shocks is necessary because time measured with block creations is prolonged as $\theta$ gets prolonged.

For the latter above, we must invent something new, because blockchain currency protocols usually include the currency supply rule, but does not have a currency absorption or write-off rule.

\subsection{Negative Interests}

In our past work [3], we have suggested an implicit inflation target. But in this paper, we propose an implementation of negative interests, and no halving rule for $V$.

Let us consider coinage eras, where each era is, for example, consecutive 2016 blocks. Hereafter in this section, time is measured by the coinage eras.

Let $Z_{t}$ be the amount of produced coins at time $t$ (if $t$ is the current coinage era, $Z_{t}$ denotes the amount of produced coins in the era so far).

We define a depreciation rule so that effective values of coins are depreciated as time elapses. This depreciation can be like $1 \%$ of the original value as of production of the coin, after every coinage era elapses. Let the amount of depreciation of coins produced at time $t$ evaluated at time $T$ to be $D_{t}(T)$.

The total supply of coins in circulation $S(T)$ at time $T$ is, then

$$
S(T)=\sum_{t=0}^{T}\left(Z_{t}-D_{t}(T)\right)
$$

which is expected to remain constant except during positive or negative demand shocks. $D_{t}(T)$ can be a function like below, in the case of $1 \%$ depreciation of the original at every coinage era.

$$
D_{t}(T)=\min \left(Z_{t}, Z_{t} \times 0.01(T-t)\right)
$$




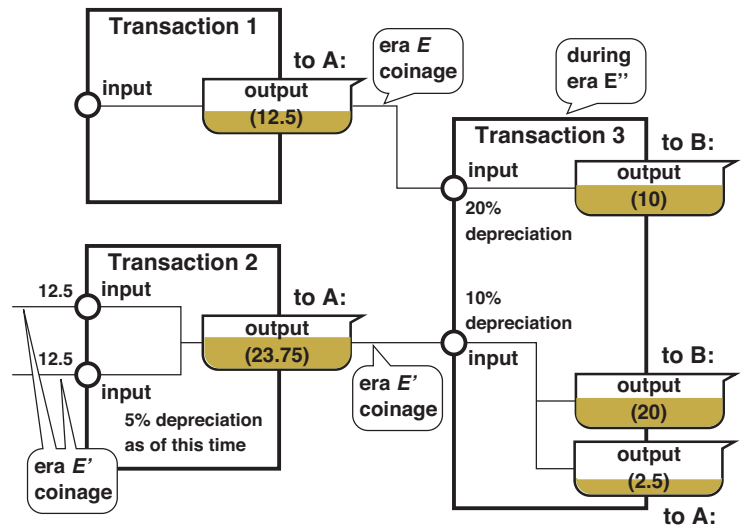

Figure 4: UTXO Transaction Structure with Depreciation of Coin Values

\subsection{Implementation for UTXO Structure}

Figure 4 shows how depreciation is implemented on a UTXO structure. In the figure, transaction 1 happened in coinage era $E$, transaction 2 happened in some coinage era between $E^{\prime}$ and $E^{\prime \prime}$, and transaction 3 is happening in coinage era $E^{\prime \prime}$.

Because coins produced in different eras have different depreciation levels, they need to be treated separately in a transaction. In transaction 3 in the figure, unlike the original design of Bitcoin transactions, inputs referring to the coin values originated from era $E$ and era $E^{\prime}$ are connected separately to different outputs to give total of value 30 to user $B$. Because coins originated at greater than or equal to 100 eras before do not exist with the $1 \%$-per-era depreciation rule (they are evaluated as value 0 , so they cannot be referred to), at-most 100 kinds of these inputs-outputs connections are expressed in a single transaction.

A series of inputs-outputs connections originated in an era forms a coinage era graph that takes a form of directed acyclic graph. In the figure, there are two of such graphs, one originated in era $E$ and another originated in era $E^{\prime}$.

To evaluate the depreciated coin value of an input, a wallet software to build a new transaction data or a miner to verify the correctness of the data does not need to look for all sources and distributions of the coinage era graph in question. It just needs to know 1) which era the graph is originated, 2) to which era the transaction being referred to belongs, and 3) the era to which the created transaction is intended to belong. For example, in the figure, to evaluate the depreciated value at era $E^{\prime \prime}$ of the output of transaction 2 , one just need to divide 23.75 with 0.95 (because of $5 \%$ depreciation at transaction 2) to get the original amount, and subtract $10 \%$ from the amount to get 22.5 .

With this depreciation rule, when it is near the border of two coinage eras, a transaction might not make it in the block of the intended era. If that happens, 
the transaction data needs to be discarded (or verification of the transaction would fail anyway because its outputs would be more than allowed), and the wallet software needs to recreate the transaction intended for the new era. Because of further depreciation in the new era, the wallet may need to reform the transaction to output the intended amount.

\section{Simulation}

First, we will make basic design checks of our proposed measures through simple simulations. Then, we will evaluate the measures using an artificial market with simulated simple human behaviors.

\subsection{Target and Reward Re-adjustments}

Figure 5 shows a sample distribution of block intervals for different total hash rates $(1 \times, 2 \times$ and $0.8 \times$ of the reference value where $\theta$ equals 10 minutes $)$ to check the validity of proposed thresholds for target and reward re-adjustments.

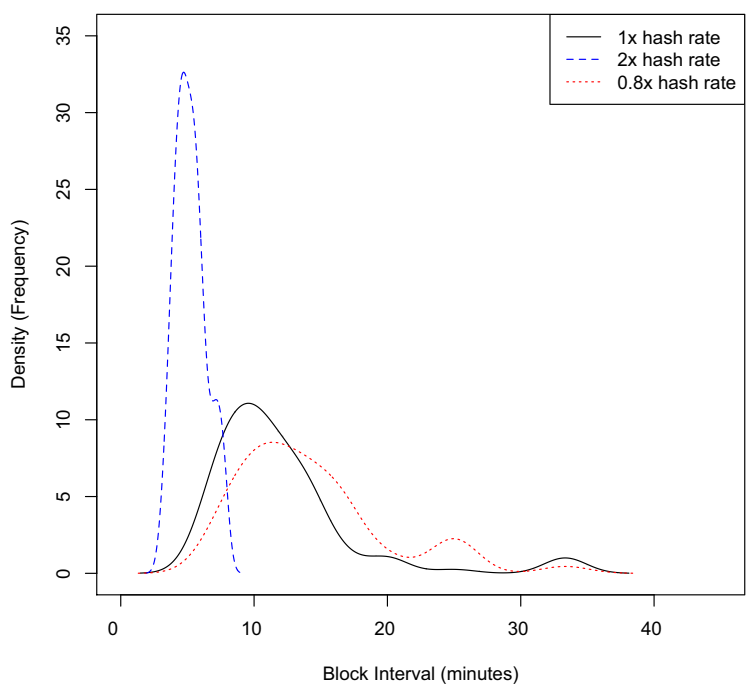

Figure 5: Distribution of Block Intervals

We assumed Poisson distribution of events. We generated 100 random counts of occurrences according to the distribution in 100 minutes, and plotted them on the graph through an evaluation of possibility density (frequency of occurrences). We have tried many iterations, and other iterations show basically the same shapes. 
In the graph, $2 \times$ of the reference hash rate produces blocks in rather short intervals. To make this well-longer than $\theta_{\min }$, the currency's network protocol may need to be improved, as suggested in [6].

On the other hand, $0.8 \times$ of the reference hash rate produces blocks in similar intervals to the reference condition. This is good in terms of detection of decreased $M$ because it means that the detection may be possible before users begin to feel that block creations are getting delayed. But do not these detections happen too often? Actual data from Bitcoin blockchain, found at 7] for example, shows that this event of the average interval reaching $\frac{5}{4}$ of the reference (12.5 minutes) is rather rare (except during congestions observed in late 2017).

\subsection{Reduction of Supply}

Figure 6 shows a simulation result on the amount of coins in circulation with depreciation. In the simulation, we have run the total of 1,000 coinage

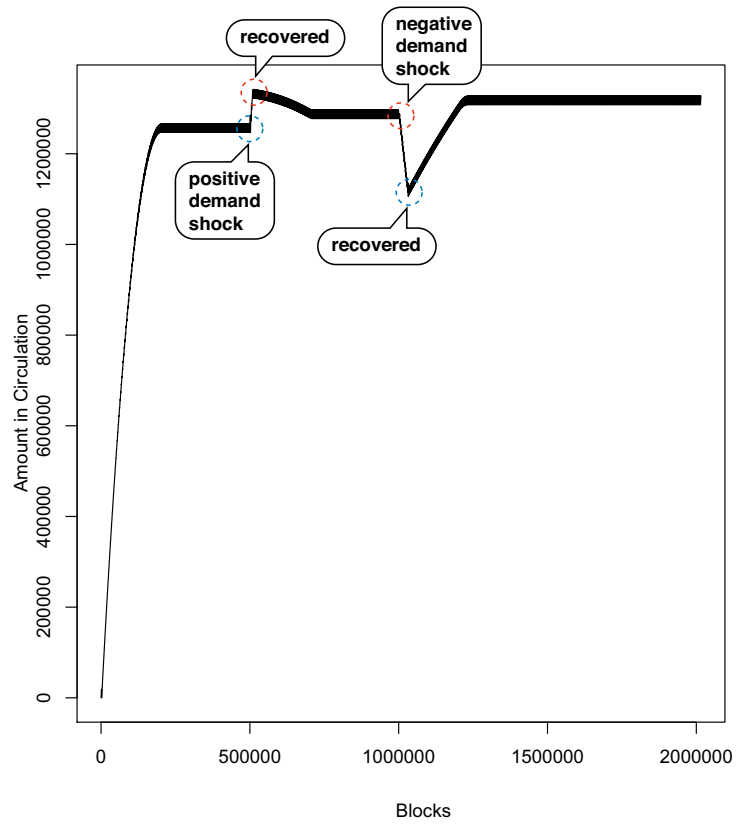

Figure 6: Simulated Total Amount in Circulation

eras each containing 2016 blocks (equals to 2,016,000 blocks). With reference block interval of 10 minutes, 100 blocks (to detect changes in $\theta$ ) amount to about 17 hours, 2016 blocks ( 1 coinage era) amount to 2 weeks, and 100 coinage eras (time for $100 \%$ depreciation) amount to a little less than 4 years. But note that the graph is drawn in blocks, not in real time. In reality, the intervals between blocks may fluctuate largely. The reward for block creation starts 
at 12.5 , and is adjusted through observed demand shocks injected artificially during the simulation.

A positive demand shock is injected at block 500,000, which is recovered around block 506,000, and a negative demand shock is injected at block 1,000,000, which is recovered around block 1,030,000.

Note that slight increase in the supply is caused by offsetting the doubling rule $(V=V \times 2)$ with taking- $80 \%$ rule $(V=V \times 0.8)$ for three times (amounts to 0.512 of $V$ at the beginning of offsetting). In reality, supply is also adjusted by autonomous fluctuation of $\theta$.

It shows that the supply of coins remains fairly constant even after positive and negative demand shocks and their recoveries by the market price mechanism.

\subsection{Artificial Market}

We call the currency with the proposed measures Stabilized Coin hereafter, and compare it with Bitcoin through simulations over an artificial market we define as follows.

\subsubsection{Model}

Agents.

We start with defining agents. We define a set of speculators $U_{s}$ where $\left|U_{s}\right|=N_{s}$ and a set of common traders $U_{r}$ where $\left|U_{r}\right|=N_{r}$. Speculators and common traders are collectively called traders hereafter. Each trader $u \in U_{s}$ or $u \in U_{r}$ is associated with market-sensitivity $u^{s}$ where $0<u^{s} \leq 1$, evenly distributed among the traders in each set. They are also associated with balance of the coins being simulated (either Bitcoin or Stabilized Coin, depending on the specific simulation) $u^{b}$, and reference price of the currency $u^{p}$ initialized as price $P$ at the beginning of each simulation. In addition, a speculator is associated with previous reference price $u^{p p}\left(u^{p}=u^{p p}\right.$ to start). We assume that they have unlimited supply of fiat currency to purchase the coins, but actual spending of the fiat currency is regulated by probabilities of making orders and ordering amounts, which are set proportional to $u^{s}$.

We define a set of mining units $U_{m}$ where $\left|U_{m}\right|=M . U_{m}$ is collectively associated with balance of the coins $U_{m}^{b}$.

Market.

We define a daily market $\left\langle U_{s}, U_{r}, U_{m}, E>\right.$ where $E$ is an exchange, at which ordering to buy or sell coins, clearing the orders, and updating the price $P$ is made once everyday.

For simplicity, miners $U_{m}$ is purely a supplier of the coins, always trying to clear $U_{m}^{b}$ by selling all coins they have.

Trader $u \in U_{s}$ or $u \in U_{r}$ makes an order to buy $u^{b} \times u^{s} \times 0.2$ amount of coins by probability $p$, and to sell the equal amount of coins by probability $1-p$ (for random market movements).

In addition, speculators and common traders behave differently as follows: 
- Speculator $u \in U_{s}$ makes an order to sell $u^{b} \times u^{s} \times 0.8$ amount of coins if the price $P$ is greater than $u^{p} \times\left(1+\frac{u^{s}}{2}\right)$, and to buy the same amount of coins if $P$ is less than $u^{p} \times\left(1-\frac{u^{s}}{2}\right)$ (they try to make profits by "buy low, sell high" strategy). In either case, $u$ updates the reference prices so that $u^{p p}:=u^{p}$ and then $u^{p}:=P$. However, in the case that $P$ is less than $u^{p p} \times\left(1-\frac{u^{s}}{2}\right) \times \frac{1}{2}, u$ makes an order to buy the same amount of coins as above, but neither $u^{p}$ nor $u^{p p}$ are updated (to save the market from crashing and to continue to make more profits).

- Common trader $u \in U_{r}$ makes an order to buy $u^{b} \times u^{s} \times 0.8$ amount of coins if the price $P$ is greater than $u^{p} \times\left(1+\frac{u^{s}}{2}\right)$, and to sell the same amount of coins if $P$ is less than $u^{p} \times\left(1-\frac{u^{s}}{2}\right)$ (they follow market trends, ending up buying high and selling low). In either case, $u$ updates the reference price so that $u^{p}:=P$.

According to the orders, matching is made at the exchange. Miners' offering is cleared first, and then the priority is set for smaller trade volumes.

\section{Price Formation.}

We adopt the simplest price formation model according to [8],

$$
\Delta P_{t}=P_{t+1}-P_{t}=\alpha\left(D_{t}-S_{t}\right)
$$

where $P_{t}, D_{t}$ and $S_{t}$ denote the price, demand and supply at time $t$ (measured by days), respectively, and $\alpha$ is the sensitivity of price against the difference between demand and supply. In the simulations, $\Delta P_{t}$ is truncated to maintain $-\frac{P_{t}}{2} \leq \Delta P_{t} \leq \frac{P_{t}}{2}$.

In reality, it is most likely that $\alpha$ is not constant. We adopt this simple model so that we can evaluate the effects of $\alpha$ over stability of the price.

\subsubsection{Parameters}

Where applicable, we use parameter values observed in the real Bitcoin blockchain and trade markets of BTC. We start our simulation time at the beginning of a reward era, namely when the reward became 12.5BTC (the start of the current reward era as of 2018). The reason behind this choice is that miners would in reality behave actively to boost the currency price upon reward-halving events, which we would like to avoid implementing in our model for simplicity. We would like to set the reward-halving event as late in the simulation time as possible.

From the information at 9], we have identified that the current reward era started on July 9, 2016. The market price in USD (US Dollar) of bitcoin on that day is found at "Market Price (USD)" section of [10], and is 652.00USD.

The estimated transaction volume on that day is found at "Estimated Transaction Value" section of [10], and is 216,546BTC. Parameters have been configured to produce more or less similar daily transaction volumes, assuming that most bitcoin transactions are trades instead of payments.

Table 1 shows the list of parameters used for the simulations. 
Table 1: Artificial Market Simulation Parameters

\begin{tabular}{|r|r|l|}
\hline Constant cost $C_{m}$ & 815 & Equation (1) is assumed to hold at start \\
Initial price $P$ & 652.00 & USD price of BTC on July 9, 2016 \\
Initial reward $V$ & 12.5 & We try 50, 25, 12.5 and 6.25 \\
Constant unit hash rate $h$ & 1.0 & Abstract rate \\
Initial target-reachability $g$ & 0.1 & Abstract probability \\
Initial number of miners $M$ & 1000 & Lower-bound is 100 \\
\hline Total coins at the beginning & $15,750,000$ & Total BTC generated before July 9,2016 \\
Number of speculators $N_{s}$ & 500 & Initial 10,000,000 coins evenly distributed \\
Number of common traders $N_{r}$ & 1,000 & Initial 5,750,000 coins evenly distributed \\
Constant probability to buy $p$ & 0.52 & To offset the tendency for larger supply \\
Constant sensitivity of price $\alpha$ & 0.0001 & We try 0.00001, 0.0001 and 0.001 \\
Simulation time in days & 3,650 & 10 years \\
\hline
\end{tabular}

Equation (11) is evaluated every day. $P V h g$ is calculated and compared with the constant cost $C_{m}$. M then increases or decreases by $1 \%$ accordingly towards the equilibrium, with its lower-bound set to be 100 .

\subsubsection{Price Changes}

Figure 7 shows the results of simulated price changes of Bitcoin and Stabilized Coin.

In reality, Bitcoin price has hit 18,962USD on December 17, 2017 as found at [10], but this simulation shows the maximum price in 10 years as just below 7,000 . We have also seen occasional drops of the price of bitcoin in reality, but the simulation shows mostly monotonous increase. By no means, this simulation is a trial to reproduce the events and their effects observed in reality, but is a demonstration of our model.

The price of Stabilized Coin seems relatively stable in the simulation, but the fluctuation may seem too big to be used as means for payment (we will later discuss this in section 6.3.6).

\subsubsection{Transacted Quantity}

Figure 8 shows the results of simulated transaction quantities of Bitcoin and Stabilized Coin, from the same simulation as the previous section.

Those begin at 261,778.7 and 259,159.92, respectively, roughly reproducing the real quantity of $216,546 \mathrm{BTC}$ as found at [10]. In the case of the simulated Bitcoin, as long as transacted quantities are concerned, the simulation seems to be a good reproduction of reality.

\subsubsection{Hash Rate}

Figure 9 shows the movements of hash rates during the same simulations of Bitcoin and Stabilized Coin.

The simulated Bitcoin hash rates do not seem real. In reality, Bitcoin hash rates grow exponentially. The reason would be that miners in reality are active 


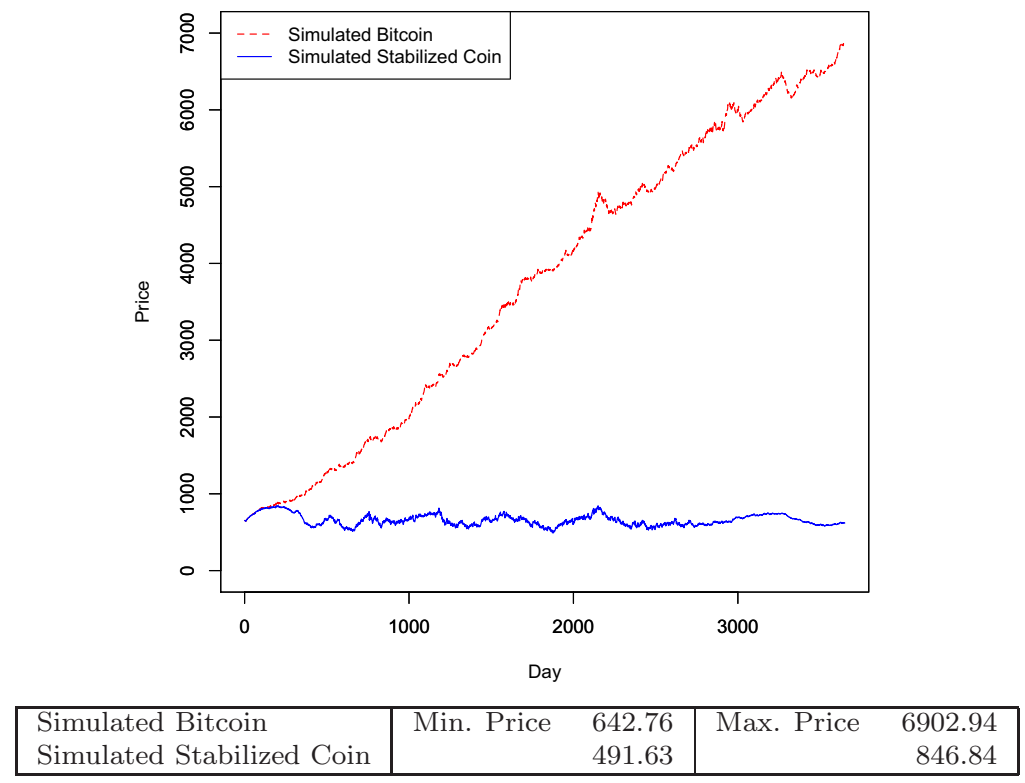

Figure 7: Simulated Price Changes of Bitcoin and Stabilized Coin

traders, and behave like speculators themselves. But instead of buying coins while their price is low, they try to produce coins by mining.

Such behaviors are not included in our model, because it would not fit in the case of Stabilized Coin, where participating in the production of coins means taking the role of lowering the currency price. We did not want to implement different agent behaviors between simulations of Bitcoin and Stabilized Coin, as it would make fair comparisons difficult.

The simulated Stabilized Coin hash rates goes up and down. The real hash rate on July 9, 2016 is found at "Hash Rate" section of [10], and was $1,580,610 \mathrm{TH} / \mathrm{s}$. Because the initial abstracted total hash rate in this simulation is 1,000 , the highest hash rate in the graph, about 30,000, would amount to $47,418,300 \mathrm{TH} / \mathrm{s}$ in reality. The observed highest Bitcoin hash rate so far (as of writing of this manuscript) was $43,161,058 \mathrm{TH} / \mathrm{s}$ on June 24,2018 . So, it seems somewhat feasible, depending on your views. However, this would raise a more serious question than just feasibility. The hash rate going up and down relatively rapidly would be a potential security threat for Stabilized Coin, discussed later in section 7.2

\subsubsection{Effects of Sensitivity of Price}

Figure 10 shows the results of simulated price changes of Bitcoin where the sensitivity of price $\alpha$ is varied among 0.001, 0.0001 (default in our model) and 


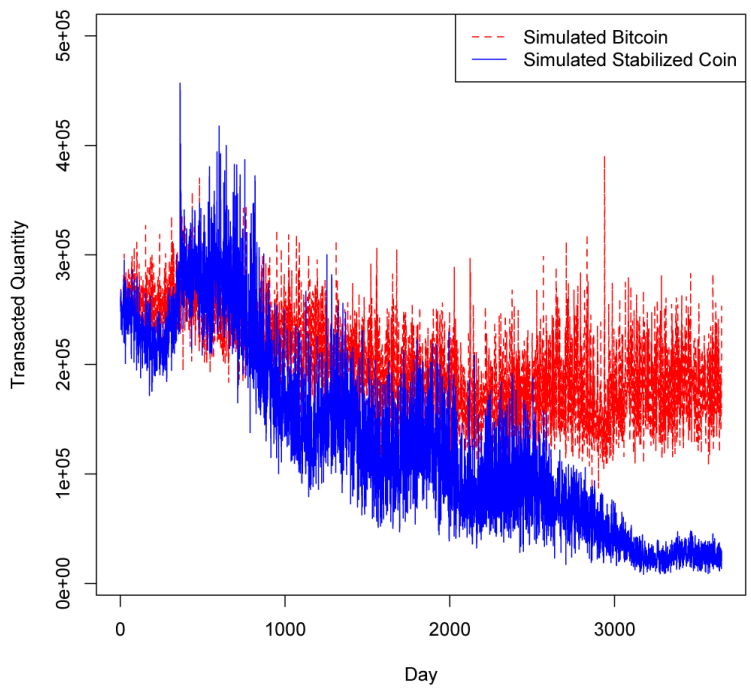

Figure 8: Simulated Transacted Quantity of Bitcoin and Stabilized Coin

0.00001 .

The effects of $\alpha$ against the simulated Bitcoin prices seem straightforward. The larger $\alpha$ is, the higher the price goes. In the case of $\alpha=0.00001$, the price seems constant in the graph, but it actually shows a mostly monotonous increase of the price, which almost doubles.

Figure 11 shows the results of simulated price changes of Stabilized Coin where the sensitivity of price $\alpha$ is varied in the same way.

It is clear from the graph that $\alpha$ takes an important role in stability of the simulated currency price. The smaller $\alpha$ is, the more stable the price becomes. But, even in the case of $\alpha=0.00001$, the price seems to fluctuate fairly largely. We need a reference to evaluate whether these levels of fluctuations are tolerable or not.

Figure 12 shows a comparison with the historical changes of USD and Japanese Yen (JPY) exchange rate. We have obtained the historical data from [1], and extracted the exchange rates of recent 10 years (July 7, 2008 to July 6, 2018). To put them in the same scale, we compared the historical changes with the prices of 0.153 Stabilized Coin (where $\alpha=0.00001$ ) and 0.151 Stabilized Coin (where $\alpha=0.0001$ ).

It shows that the price changes of the simulation results are comparable with the price changes of currencies in real life, although fluctuation is larger in the case of $\alpha=0.0001$. 


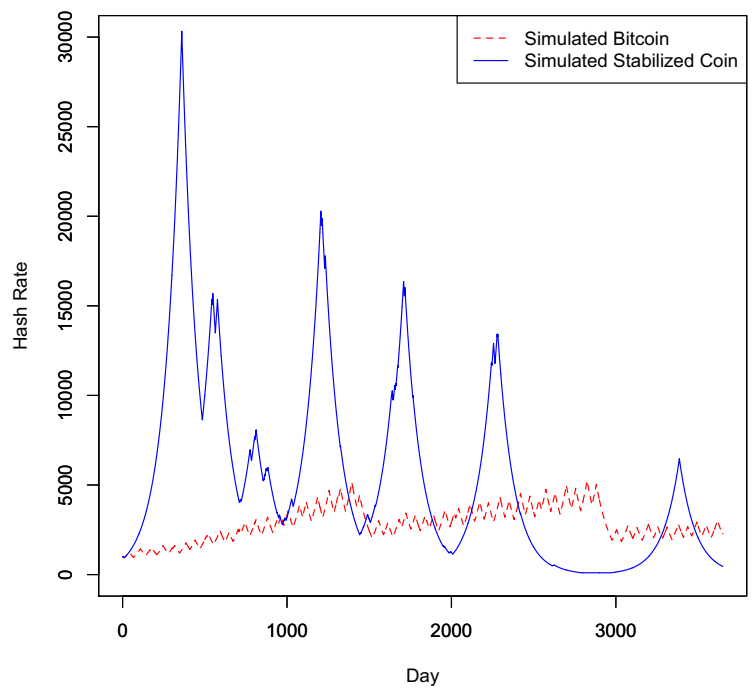

Figure 9: Simulated Hash Rates of Bitcoin and Stabilized Coin

\subsubsection{Effects of Initial Rewards}

Figure 13 shows the results of simulated price changes of Stabilized Coin where the initial reward for making blocks is varied among 6.25, 12.5 (default in our model), 25 and 50.

It shows that the smaller the initial reward is, the higher the price tends to go, because the rewards at block creations represent new supply to the market, bringing the price of coins down.

In the case of initial reward $=25$, the graph shows that the price goes stable while slowly declining after around day 2,000. We do not think that this is a success, but instead, it shows limitation of our simulation model. In this particular case, the distribution of the coins makes that random market movements cannot be large enough to cause speculative moves of traders any more.

\section{Discussion}

\subsection{Responsiveness}

In order for the price stabilization to work, the number of miners $M$ needs to be quickly responding to demand changes. But the initial investment cost and sunk cost effects for miners may defer their entries and exits, although exiting miners have a choice of just turning (part of) their machines off instead of entirely leaving the business. 


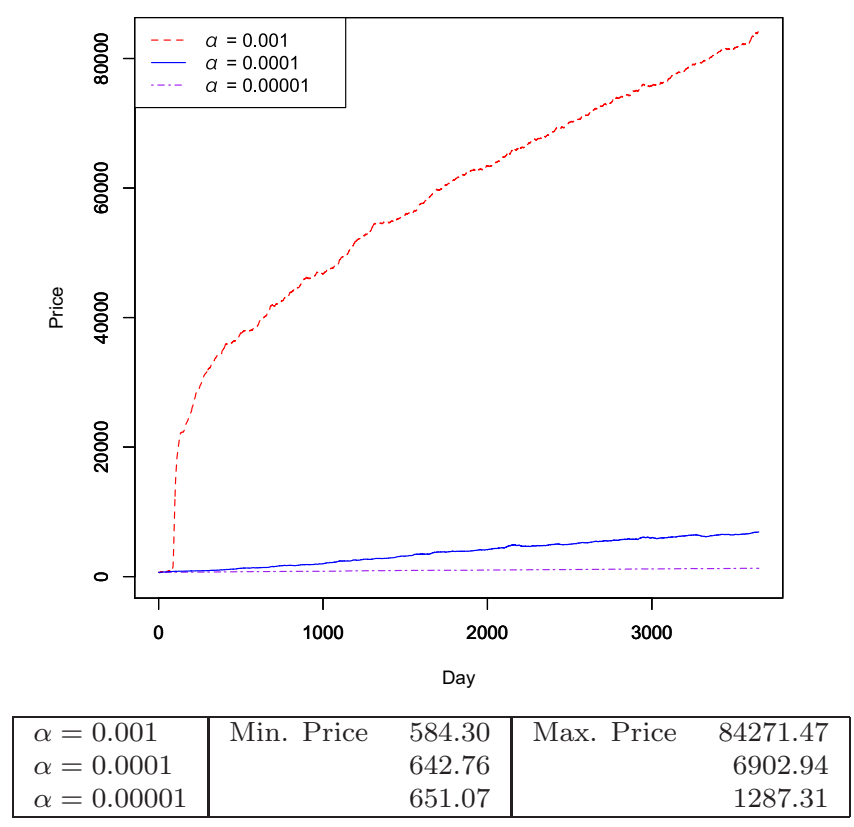

Figure 10: Effects of Sensitivity of Price $\alpha$ (Bitcoin)

Recently, some new blockchain currencies have been started by hard forks (backward-incompatible changes of the protocols) from existing currencies. It is called a split of a blockchain.

Ethereum Classic [12], started in 2016, is probably the first of such instance, splitted from Ethereum due to a disagreement in the governance. Bitcoin Cash [13], started in 2017, is the first instance of splitting a new currency from Bitcoin.

Unless further changes are made on the proof of work algorithm, mining these currencies is compatible with the original blockchain currency. Therefore, miners may switch back and forth among compatible currencies, pursuing more profits. This switch can even be automated. With this competition among currencies, $M$ can be quickly responding to price changes according to demand shocks, making the proposed measures more effective.

\subsection{Security}

However, variance of $M$ would raise security concerns. A blockchain based on proof of work is protected by the collective cost of mining. Bitcoin blockchain is difficult to attack because its hash rate is exponentially growing, so that existing mining facilities become obsolete quickly. If it is not the case, by utilizing 


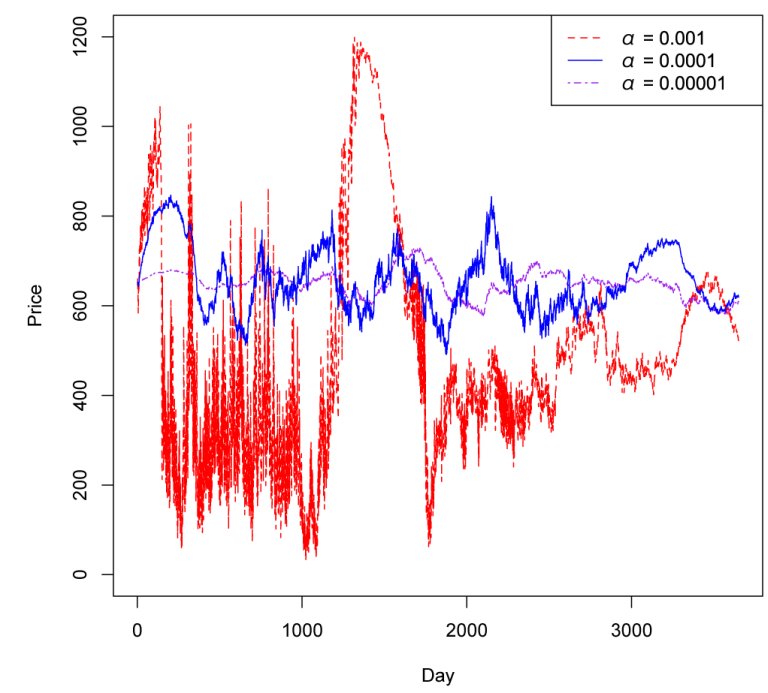

\begin{tabular}{|l|rr|rr|}
\hline$\alpha=0.001$ & Min. Price & 31.96 & Max. Price & 1198.52 \\
$\alpha=0.0001$ & & 491.63 & & 846.84 \\
$\alpha=0.00001$ & & 576.46 & & 728.39 \\
\hline
\end{tabular}

Figure 11: Effects of Sensitivity of Price $\alpha$ (Stabilized Coin)

available unused facilities, it would be easier to conduct so-called $51 \%$ attack 3 or block withholding attack (variance of selfish mining [14] to withhold created blocks while broadcasting contradicting transactions to perform double spending). This type of threat is real in many existing cryptocurrencies, with actual cases of attacks against currencies called Monacoin and Bitcoin Gold 15] in May 2018. This problem seems to be inherent of the design based on proof of work.

\subsection{Applicability to Structures other than UTXO}

We described how to implement depreciation, or negative interests, on a UTXO structure in section 5.3. But there are blockchain currencies that do not depend on UTXO structures. Ethereum, for example, maintains the account states in each participating node, and transactions (encoded state transfers) only are stored in the blockchain.

With this account-state design approach, assuming the rule of $1 \%$ depreciation over a coinage era we described, 100 different sets of balances are required for an account to maintain a single currency, for storing the values without

\footnotetext{
${ }^{3}$ Actually, attack should be meaningful upon reaching just $50 \%$ of total hash rate, in which case it becomes uncertain whether the correct miners would win the race of creating blocks or not.
} 


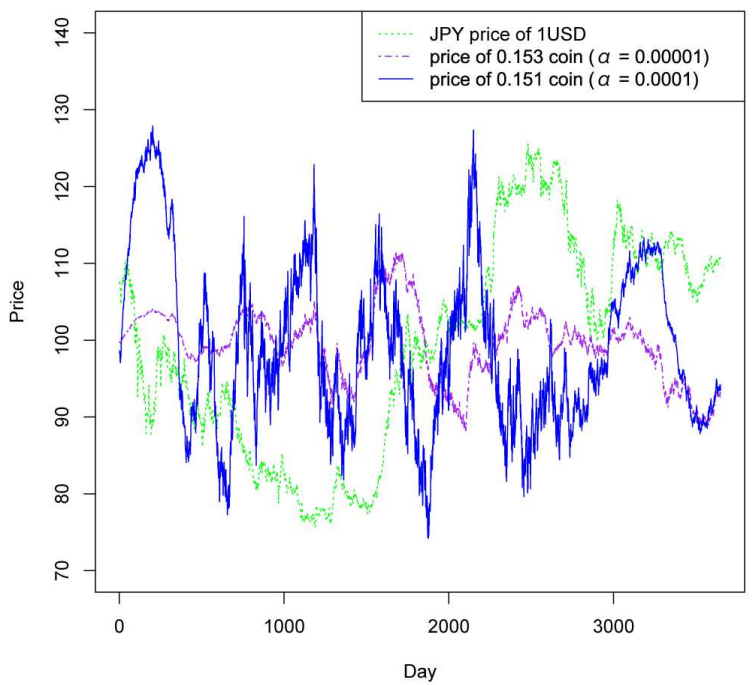

\begin{tabular}{|l|ll|rr|}
\hline JPY price of 1USD & Min. Price & 75.72 & Max. Price & 125.58 \\
price of 0.153 coin $(\alpha=0.00001)$ & & 88.20 & & 111.44 \\
price of 0.151 coin $(\alpha=0.0001)$ & & 74.24 & & 127.87 \\
\hline
\end{tabular}

Figure 12: Comparison with Historical Changes of USD-JPY Exchange Rate

depreciation in the past 100 eras separately. These values are each negatively weighted as they are depreciated, upon calculating the effective balances.

\subsection{Deployment}

If we start a new blockchain currency with the proposed measures from its genesis block, it would have to start with virtually zero price level, and since the mechanism is intended to avoid speculations, the price level would remain near zero, not suitable to be used as a monetary medium to represent values of goods or services.

To avoid such a situation, we may want to utilize a split to employ the inertia of the original existing currency. If we start a new currency by splitting it from Bitcoin, for example, the new currency can start with a useful price level and enough attention, although how existing coins should be depreciated is a problem to consider. In the artificial market simulation in section 6.3. we just implemented depreciation in a straightforward way, not considering human reactions.

\subsection{Sustainability}

With the proposed measures, the amount of coins in circulation is automatically maintained to be near constant, and there is no need for external measures 


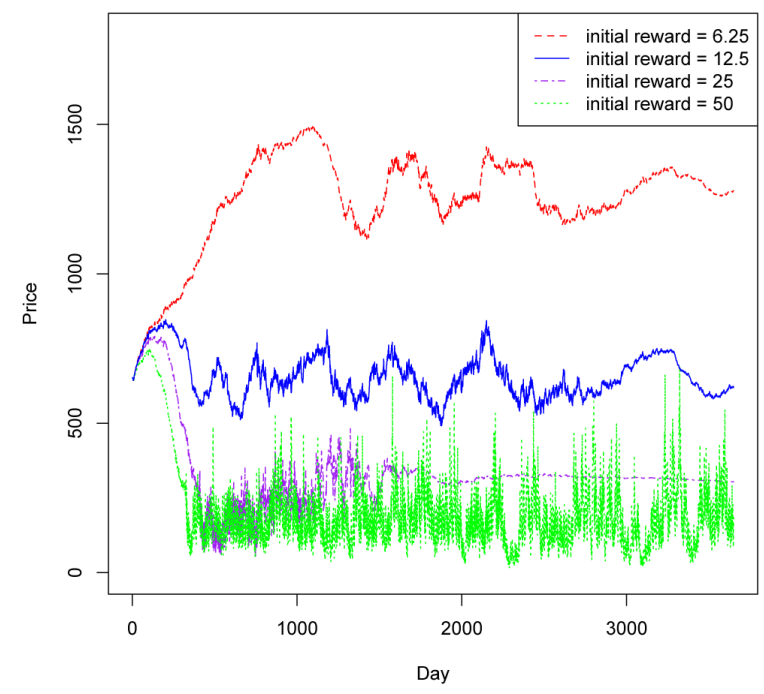

Figure 13: Effects of Initial Rewards (Stabilized Coin)

to cap the supply of coins, such as the rule of halving rewards as found in Bitcoin. Rewards for block creations to miners can be perpetual, providing a possible solution to the problem of sustainability in concern.

This means that miners do not need to rely on fees, so we may as well want to abandon transaction fees altogether.

\subsection{Effects of Depreciation}

In order for the price stabilization to work, the created coins must actually be supplied into the market. In our proposal, because coins are depreciated, miners are incentivized to sell coins they created before they lose values. The supply is expected to be quick.

But this should be true for all users, not only for miners. In other words, depreciation should accelerate spending. The first author of this paper has made a detail study on the effects of depreciating (and amplifying) digital currencies in [16] before rise of blockchain currencies. Some of the outcomes of the work may still be applicable.

But a natural question would be, do people prefer currencies that depreciate over others that do not? Our answer may be non-intuitive, but yes.

In our proposal, transactions can be free of charge, as suggested in the previous section. Then depreciation can be a beneficial choice for users, because choices are between always paying transaction fees and possibly avoiding any 
kinds of it by spending before depreciation takes place. (Note that we are discussing digital currencies as media of exchange instead of investment products.)

\subsection{Effects of Splitting}

Finally, let us consider the case where a currency with proposed measures itself gets splitted, as it is a possibility for any blockchain currencies today.

If it happens, it creates a situation where $M$ decreases for both original and newly splitted currencies. Thus, both currencies would automatically react as if a negative demand shock occurred (which may be the case anyway, if the same set of budgets is used for purchasing both currencies). In case the new currency took half of the miners, the pace of block creation is slowed down, but eventually the average interval $\theta$ gets normal, and the reward $V$ is about halved for both currencies. If these two currencies compete well, the total coin amount in circulation is eventually halved for each currency, maintaining the total sum of the amounts of two currencies to be equal to the amount of the original currency coins in circulation before the split. When the process is complete, all coins copied to the new currency upon the split would have already been depreciated down to zero, and the two currencies are completely independent from one another in terms of the coins in circulation.

Therefore, in theory, the split would eventually result in two currencies with the maintained same level of prices as before and with the same amount in total in circulation as before. But how the market in reality reacts against this would need further study.

\section{Related Work}

\subsection{Improvements to Digital Currency Design}

Improvements to the original design of Bitcoin have been proposed many times. Many of them have taken the forms of alternative coins (altcoins), instead of research papers.

Among these, one of the most well-known examples is Litecoin 17], from which many other altcoins forked their code. Litecoin has shown that with basically the same proof of work mechanism as Bitcoin (although cryptographic hash function in question is different), average block intervals can be as short as 2 minutes (Ethereum maintains average block intervals as short as 15 seconds, but with lots of other improved designs).

Bitcoin Cash was born by a split from Bitcoin, addressing the issue of improving the throughput by adjustable block sizes. Many other new currencies have followed the practice of splitting.

Sidechains 18 provide another forms of testing improvements to blockchains and their currencies.

To our best knowledge, none of these address measures against the negative demand shock (no means to collect coins in circulation to control supply of them). 
Another approach towards stabilization of currency prices is stable coin [19], which is relatively a new term, but the concept has existed for many years in the form of electronic money. The prices of the coins are pegged with fiat currencies. Tether 20] is a well known example of such stable coins.

\subsection{Models of Economics}

Economics of mining has also been modeled in [21] 222], where the model is used for simulating the price clearing mechanism in the market using a virtual order book similar to that presented in 23]. Another trial on understanding economics of Bitcoin through simulations with an artificial market is found in [24], using the terminology and strategies of actual tradings. 25] makes research on competition among cryptocurrencies. [26] analyzes how Bitcoin prices were historically formed through exchanges. [27] and 28] each gives a view of Bitcoin Economics. 29] gives such a view on cryptocurrencies in general.

Our model is far simpler compared with these, intended for analysis of the problems and devising solutions. The points of our work are twofold: 1) by paying attention to economically rational behaviors of miners, where mining can be viewed as if it is more or less like production of goods in firms, we can describe how miners behave against coin prices in perspective, and 2) by changing the rules for such behaviors of miners, we can create a negative feedback to the coin prices.

We understand that there may be a different approach, e.g., to formulate a total economics such that the coin prices arise endogenously from the model. We agree that such a model, if successfully built, has significance. However, we believe that it is difficult to fulfill our goal with such an approach, because any price is rational in Bitcoin-like currencies - whatever a price may be, once it is predicted that the market would hold it, then the difficulty target for producing coins will be adjusted accordingly to the price level, so that any price is effectively an equilibrium price that balances the supply and demand. In other words, the prices are determined by the psychological states of those who are interested in the coins. It means that the prices can easily be manipulated. If we could build a model from which the coin prices arise endogenously, people would still be able to make movements that would break the model. Instead of working with such a difficulty of handling psychological states of people, our method is to begin with the view that miners reacting against prices resemble firms producing goods, and we believe that we have shown that still it can lead to fulfilling our goal of designing a system that would make the currency prices stable.

The objective of equation (11) is to formulate behaviors of miners, instead of constructing a total economic model for coin prices. We believe that we have successfully formulated the behaviors of miners, as our model reflects the reality.

When we see the actual movements of prices and hash rates of Bitcoin 4 , we observe general long-term tendencies that the hash rate rises as the price keeps

\footnotetext{
${ }^{4}$ Observable from, for example, https://www.blockchain.com/charts
} 
rising (the all-time tendency of Bitcoin hash rate until September 2018), and the hash rate falls as the price keeps falling (a tendency of Bitcoin hash rate from September to December 2018 - a downfall tendency never observed before). Miners can freely behave speculatively to try to maintain or raise the values of the coins they own in the short term, but in the long run, as we have predicted, their moves seem to conform to the economic rationality of equation (1).

However, in order to evaluate the effectiveness of our proposed measures in more detail, and to further improve the design, we may want to refine the artificial market methods according to those found in related work above ourselves, with insights from past work on dynamic behaviors of prices such as [30], and on agent-based simulations such as [31].

\section{Conclusions}

Continuing from our past work, this paper proposed minimal changes to the design of blockchain currencies so that their market prices are automatically stabilized, absorbing both positive and negative demand shocks of the currencies by autonomously controlling their supplies.

We have evaluated the proposed measures with simple simulations including those using an agent-based artificial market. We have shown that resulted price changes are comparable with those of currencies in real life, suggesting the effectiveness of the measures. However, more sophistications of the simulations and/or testbed trials would be needed to further the research.

In addition to stabilization of prices, the proposed measures may have effects of making those currencies preferred means for payment by disincentivizing hoarding, and improving sustainability of the currency systems by making rewards to miners perpetual without need for transaction fees.

\section{References}

[1] S. Nakamoto, Bitcoin: A Peer-to-Peer Electronic Cash System, http://bitcoin.org/bitcoin.pdf (2008).

[2] V. Buterin, A Next-Generation Smart Contract and Decentralized Application Platform, https://github.com/ethereum/wiki/wiki/White-Paper (2014).

[3] M. Iwamura, Y. Kitamura, T. Matsumoto, K. Saito, Can we stabilize the price of a cryptocurrency?: Understanding the design of bitcoin and its potential to compete with central bank money, Hitotsubashi Journal of Economics Vol.60 (1).

[4] Bitcoin Project, Bitcoin resources, https://bitcoin.org/en/resources (as of January 2018). 
[5] K. Saito, H. Yamada, What's so different about blockchain? - blockchain is a probabilistic state machine, in: IEEE 36th International Conference on Distributed Computing Systems Workshops (ICDCSW), 2016, pp. 168175 .

[6] C. Decker, R. Wattenhofer, Information propagation in the bitcoin network, in: IEEE P2P 2013 Proceedings, 2013, pp. 1-10.

[7] BitInfoCharts, Bitcoin Block Time chart, https://bitinfocharts.com/comparison/bitcoin-confirmationtime.html (as of June 2018).

[8] B. LeBaron, A builder's guide to agent-based financial markets, Quantitative Finance Vol.1 (2).

[9] bitcoin wiki, Controlled supply, https://en.bitcoin.it/wiki/Controlled_supply (as of June 2018).

[10] Blockchain Luxembourg S.A.R.L, Blockchain Charts, https://www.blockchain.com/en/charts (as of June 2018).

[11] Macrotrends LLC, Dollar Yen Exchange Rate (USD JPY) - Historical Chart, http://www.macrotrends.net/2550/dollar-yen-exchange-rate-historicalchart (as of June 2018).

[12] Ethereum Classic, Ethereum Classic, https://ethereumclassic.github.io (as of January 2018).

[13] bitcoincash.org, Bitcoin Cash - Peer-to-Peer Electronic Cash, https://www.bitcoincash.org/en/ (as of January 2018).

[14] I. Eyal, E. G. Sirer, Majority is not enough: Bitcoin mining is vulnerable, Communications of the ACM Vol.61 (7).

[15] Joseph Young, Bitcoin Gold Hit by Double Spend Attack, Exchanges Lose Millions, https://www.ccn.com/bitcoin-gold-hit-by-double-spend-attack-exchangeslose-millions/ (as of June 2018).

[16] K. Saito, E. Morino, The brighter side of risks in peer-to-peer barter relationships, Future Generation Computer Systems Vol.26 (8).

[17] Litecoin Project, Litecoin - Open source P2P digital currency, https://litecoin.org/ (as of January 2018).

[18] A. Back, M. Corallo, L. Dashjr, M. Friedenbach, G. Maxwell, A. Miller, A. Poelstra, J. Timón, P. Wuille, Enabling Blockchain Innovations with Pegged Sidechains, https://blockstream.com/sidechains.pdf (2014). 
[19] S. Lee, Explaining stable coins, the holy grail of cryptocurrency, Forbes (March 2018).

[20] Tether, Tether, https://tether.to (as of June 2018).

[21] L. Cocco, M. Marchesi, Modeling and simulation of the economics of mining in the bitcoin market, PLoS ONE Vol.11 (10).

[22] L. Cocco, G. Concas, M. Marchesi, Using an artificial financial market for studying a cryptocurrency market, Journal of Economic Interaction and Coordination Vol.12 (2).

[23] M. Raberto, S. Cincotti, C. Dose, S. M. Focardi, M. Marchesi, Price formation in an artificial market: limit order book versus matching of supply, Lecture Notes in Economics and Mathematical Systems Vol.550.

[24] Y. Shimada, A modelling of bitcoin price dynamics by the artificial market algorithm, 3072304, SSRN (October 2017).

[25] N. Gandal, H. Hałaburda, Competition in the cryptocurrency market, Working Paper 2014-33, Bank of Canada (August 2014).

[26] M. Brandvold, P. Molnár, K. Vagstad, O. C. A. Valstad, Price discovery on bitcoin exchanges, Journal of International Financial Markets, Institutions \& Money Vol.36.

[27] S. Athey, I. Parashkevov, V. Sarukkai, J. Xia, Bitcoin pricing, adoption, and usage: Theory and evidence, Working Paper 17-033, Stanford Institute for Economic Policy Research (SIEPR) (August 2016).

[28] L. Schilling, H. Uhlig, Some simple bitcoin economics, Working Paper 201821, Becker Friedman Institute for Economics at UCHICAGO (March 2018).

[29] W. Bolt, M. R. van Oordt, On the value of virtual currencies, Staff Working Paper 2016-42, Bank of Canada (August 2016).

[30] A. Beja, M. B. Goldman, On the dynamic behavior of prices in disequilibrium, Journal of Finance Vol.35 (2).

[31] M. Raberto, S. Cincotti, S. M. Focardi, M. Marchesi, Agent-based simulation of a financial market, Physica A: Statistical Mechanics and its Applications Vol.299 (1-2). 